\title{
楔形螺纹连接扭拉关系理论分析及拧紧特性 ${ }^{*}$
}

\author{
邓新建 ${ }^{1}$ 刘检华 ${ }^{1}$ 张忠伟 $^{2}$ 巩 浩 $^{1}$ \\ (1. 北京理工大学机械与车辆学院 北京 100081; \\ 2. 中国航天标准化研究所 北京 100166)
}

\begin{abstract}
摘要: 楔形锁紧螺母是一种应用广泛的防松结构, 但是当前关于楔形螺纹连接的扭拉关系理论分析和拧紧特性因素分析的研 究成果匮乏, 导致喫形锁紧螺母的安装工艺一直缺少理论指导。首次建立并验证了楔形锁紧螺母拧紧过程的扭拉关系理论公 式, 并对其拧紧特性进行了影响因素分析。首先考虑楔形螺纹面的复杂几何形状, 构建楔形斜面上任意区域的局部坐标系, 通过坐标转换获得了任意楔形区域的平面方程、切向和法向矢量, 在此基础上建立楔形锁紧螺母拧紧过程的扭拉关系理论公 式; 然后建立精确的楔形螺纹连接有限元模型, 仿真了实际的拧紧过程, 通过改变不同的摩擦系数、楔形角和螺距, 得到拧 紧扭矩-预紧力的离散数据点, 通过对比理论公式所绘制的扭拉关系直线, 从数值仿真角度验证了楔形螺纹连接扭拉关系理论 公式的可靠性; 同时, 采用航空航天领域的两种典型楔形锁紧螺母作为试验对象, 通过拧紧试验进一步验证理论公式的可靠 性。最后系统研究了螺栓螺母材料、被压件材料、螺纹规格、螺母结构形式、拧紧次数等因素对楔形锁紧螺母扭矩系数和重 复拧紧性能的影响规律。
\end{abstract}

关键词: 楔形锁紧螺母; 扭拉关系; 有限元仿真; 扭拉试验; 重复拧紧性能

中图分类号: TG156

\section{Theoretical Analysis on Torque-tension Relationship and Tightening Characteristics of Wedge Threaded Connection}

\author{
DENG Xinjian $^{1}$ LIU Jianhua ${ }^{1}$ ZHANG Zhongwei ${ }^{2}$ GONG Hao ${ }^{1}$ \\ (1. School of Mechanics and Vehicles, Beijing Institute of Technology, Beijing 100081; \\ 2. China Aerospace Standardization Institute, Beijing 100166)
}

\begin{abstract}
Wedge self-locking nut is a widely used anti-loosening structure. However, currently, there is a lack of theoretical analysis on the torsion-tension relationship and tightening characteristics of wedge threaded connection, which leads to the lack of theoretical guidance on the installation of wedge self-locking nut. The theoretical formula of torsion-tension relationship during the tightening process of wedge self-locking nut is established and verified for the first time, and the influencing factors of the tightening characteristics of wedge threaded connection are analyzed. First, the complex geometry of the wedge threaded surface is considered, the local coordinate system of any region on the wedge thread surface is constructed, and the plane equation, tangential vector and normal vector of any wedge area are obtained by coordinate transformation. On this basis, the theoretical formula of the torsion-tension relationship of wedge self-locking nut is established. Second, the accurate finite element model of wedge threaded connection is established and the actual tightening process is simulated. The different discrete data points of torque-tension are obtained by changing different friction coefficients, wedge angles and pitches. These data points of torque-tension are then compared with the relationship curve of torque-tension described by the theoretical formula, based on which the reliability of the theoretical formula of torque-preload is verified. At the same time, two typical wedge self-locking nuts in aerospace field are used as test objects, and the reliability of the theoretical formula is further verified by tightening experiment. Finally, the influences of the material of bolt and nut, the material of joint, the thread specification, the structural form of nut and the number of tightening times on the torque coefficient and the repeated tightening performance of the wedge self-locking nut are studied systematically.
\end{abstract}

Key words: wedge self-locking nut; torque-tension relationship; finite element simulation; torque-tension experiment; repeated tightening performance

* 国家自然科学基金资助项目(51935003；51675050)。20201101 收到初稿, 20210210 收到修改稿 


\section{0 前言}

螺纹连接具有拆卸方便、互换性强、造价低廉、 可重复使用等优点, 在各种机械产品中应用广泛。 机械产品在服役的过程中, 很可能暴露在振动、冲 击等恶劣环境中, 或者机械产品本身一直处于振动 状态, 使得螺纹连接很容易出现松动失效 ${ }^{[1-7]}$, 从而 降低机械产品可靠性, 甚至引发严重的安全事故 ${ }^{[8]}$ 。 为了提高螺纹连接的防松性能, 工程中经常应用一 些防松结构 ${ }^{[9-14]}$, 例如双螺母、哈德洛克螺母、弹 簧垫圈、齿形垫圈等。其中, 楔形锁紧螺母通过改 变内螺纹的牙型结构, 在和普通螺栓配合使用时表 现出了优异的防松效果, 并大量应用在铁路、车辆、 航空航天等领域。

国外学者和研究机构对楔形锁紧螺母的防松性 能进行了系统评估 ${ }^{[15]}$, 例如美国宇航局戈达德太空 飞行中心的科研人员对比了楔形锁紧螺母和普通螺 母在振动条件下的防松效果, 研究结果表明, 楔形 锁紧螺母可以一直保持紧固状态直到螺栓发生疲劳 失效, 而普通螺母则在振动开始的几分钟以后就出 现了松动; 美国德纳公司通过冲击耐久性试验测试 发现, 楔形锁紧螺母的防松性能甚至比螺纹锁固胶 还要高 $15 \% \sim 20 \%$ 。最近几年, 国内学者也对楔形 锁紧螺母的防松性能进行了广泛而深入的研究。李 作霖等 ${ }^{[16]}$ 利用振动试验台开展了普通螺母和楔形 锁紧螺母的防松性能对比测试, 研究结果表明, 普 通螺母在 $14 \mathrm{~min}$ 以后就出现了松脱, 而楔形锁紧螺 母则在 $180 \mathrm{~min}$ 以后才出现轻微松动; 张翼等 ${ }^{[17]}$ 选 取了 4 种适合航天产品使用的紧固件防松方式, 即 打冲点、双螺母、弹簧垫圈和楔形锁紧螺母, 对紧 固件实施冲击加速振动试验, 结果发现, 相较于传 统的防松方式, 楔形锁紧螺母表现出了更优异的防 松能力。杨柳等 ${ }^{[18]}$ 建立了一套可靠性评估流程以及 可靠性理论模型, 并开展了楔形锁紧螺母的可靠性 评估与分析。余威等 ${ }^{[19]}$ 证明了楔形锁紧螺母在 50 次重复拆装、极限交变温度等条件下仍然具有很好 的抵抗振动松脱的能力。GONG 等 ${ }^{[20]}$ 提出了评估楔 形锁紧螺母防松性能的优化方法, 试验和仿真结果 均表明, 具有 $30^{\circ}$ 楔形角的楔形锁紧螺母具有最优 的防松性能。李天雷等 ${ }^{[21]}$ 通过振动试验和仿真分析 发现, 为了保证楔形锁紧螺母的优异防松性能, 初 始预紧力不能过大也不能过小。

除了防松性能以外, 学者们还对楔形锁紧螺母 的拧紧特性进行了研究。TREMSIN 等 ${ }^{[22]}$ 指出, 在
产生相同预紧力的情况下, 楔形锁紧螺母所需的拧 紧扭矩比普通螺母需要的拧紧扭矩高 10\% $20 \%$ 。魏 天赐等 ${ }^{[23]}$ 通过扭拉试验发现, 在施加相同装配扭矩 的情况下, 楔形锁紧螺母所获得的轴向力 (即预紧力) 比普通螺母所获得的轴向力低 $10 \%$ 以上。张翼等 ${ }^{[17]}$ 同样证明了楔形锁紧螺母的扭矩系数比普通螺母 高。此外, 余威等 ${ }^{[19]}$ 还通过研究发现楔形锁紧螺母 的扭矩系数随安装力矩的增大而逐渐增大, 然后趋 于稳定，并计算了其在正常使用条件下的最小安装 扭矩。

从上述的研究中可以看出, 在过去的研究工作 中, 学者们对楔形锁紧螺母的防松性能进行了大量 研究, 取得了丰富的研究成果, 证明了楔形锁紧螺 母优异的防松性能, 并揭示了其防松原理和使用条 件。但是, 关于楔形螺纹连接的扭拉关系理论分析 和拧紧特性因素分析的研究成果仍然贵乏, 当前仅 仅通过扭拉试验发现楔形锁紧螺母比普通螺母更难 拧紧, 即扭矩系数更大, 但缺少针对楔形螺纹连接 拧紧过程的理论分析, 尚无准确定量的扭拉关系理 论公式, 用于指导楔形锁紧螺母的实际拧紧过程, 而且, 各种因素条件下, 楔形锁紧螺母的扭矩系数 和重复拧紧以后的紧固特性也需要进一步研究分 析。因此, 首先构建楔形斜面上任意区域的局部坐 标系, 通过坐标转换获得了任意楔形区域的平面方 程、切向和法向矢量, 建立楔形锁紧螺母拧紧过程 的扭拉关系理论公式。接着, 建立精确的楔形螺纹 连接有限元模型, 仿真实际的拧紧过程, 得到扭拉 关系, 通过改变不同的参数仿真验证理论公式的可 靠性; 同时, 对航空航天领域的两种典型楔形锁紧 螺母开展拧紧试验, 通过试验进一步验证理论公式 的可靠性。最后, 试验研究螺栓螺母材料、被压件 材料、螺纹规格、螺母结构形式、拧紧次数等因素 对楔形锁紧螺母扭矩系数和重复拧紧性能的影响 规律。

\section{1 楔形螺纹连接拧紧过程的理论分析}

楔形螺纹连接是指楔形锁紧螺母和普通螺栓配 合使用的连接形式, 其内外螺纹牙剖视图如图 1a 所示, 图 $1 b$ 是普通螺纹连接的内外螺纹牙剖视图, 通过对比可以发现, 相比于普通内螺纹, 楔形螺纹 的特别之处在于其螺纹牙底部有一个 $30^{\circ}$ 的楔形 斜面(见图 1a), 在楔形锁紧螺母拧紧的过程中, 外 螺纹的牙尖挤压楔形斜面, 内外螺纹之间形成线接 触配合。一方面, 牙尖和楔形斜面的线接触配合产 
生了更大的径向压力; 另一方面, 牙尖容易变形, 使得载荷均匀地分布在接触的螺旋线上 ${ }^{[24-25]}$, 这些 都有助于提高楔形锁紧螺母的防松能力。

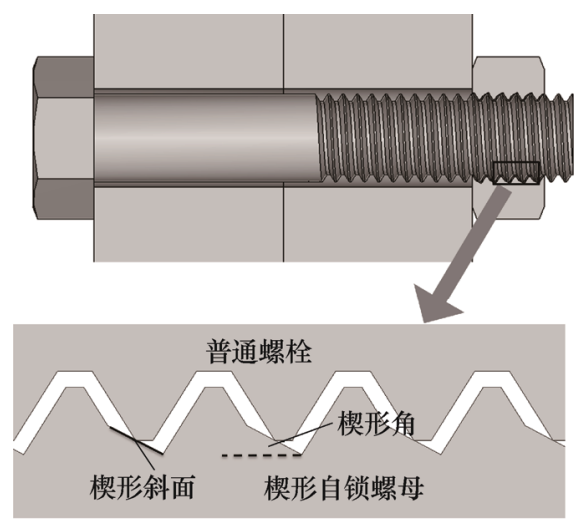

(a) 楔形螺纹连接

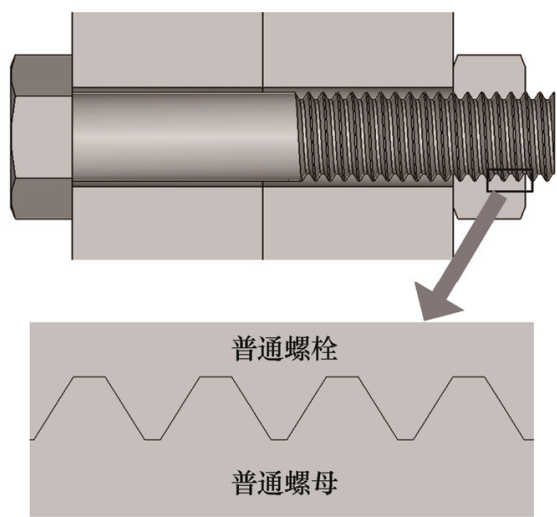

(b) 普通螺纹连接

图 1 楔形螺纹连接和普通螺纹连接的螺纹牙剖视图

为了对楔形螺纹连接的拧紧过程进行理论分 析, 首先需要对楔形斜面的几何形状进行表征, 利 用微小的矩形平面 $O B C D$ 表征楔形斜面上的某一任 意区域。楔形斜面 $O B C D$ 的产生过程如图 2 所示, 建立局部坐标系 $(x, y, z)$, 位于平面 $Y O Z$ 上的原始矩 形 $O B_{1} C_{1} D_{1}$ 将通过两次旋转得到楔形斜面 $O B C D$, 如图 $2 \mathrm{~b}$ 所示, 先固定点 $O$ 和 $B_{1}$, 将矩形 $O B_{1} C_{1} D_{1}$ 沿轴线 $O B_{1}$ 顺时针旋转角度 $\gamma, \gamma$ 表示楔形角, 得到 矩形 $O B_{2} C_{2} D_{2}$; 接着固定点 $O$ 和 $D_{2}$, 将矩形 $O B_{2} C_{2} D_{2}$ 沿轴线 $O D_{2}$ 向上旋转角度 $\beta, \beta$ 表示螺旋升角, 最终 得到楔形斜面 $O B C D$, 如图 $2 \mathrm{~d}$ 所示。 $\boldsymbol{w}$ 和 $\boldsymbol{v}$ 分别表 示垂直于楔形斜面的法向矢量和楔形螺纹拧紧的摩 擦切向向矢量如图 2c 所示, $\boldsymbol{i} 、 \boldsymbol{j} 、 \boldsymbol{k}$ 分别表示 $x 、 y$ 、 $z$ 方向的单位矢量。假设矩形 $O B C D$ 的大小是 $1 \times 1$, 经过第一次旋转以后(图 2b), $O 、 B_{2} 、 C_{2}$ 和 $D_{2}$ 的坐 标分别变成 $O(0,0,0) 、 B_{2}(0,1,0) 、 C_{2}(\sin \gamma, 1, \cos \gamma)$ 和 $D_{2}(\sin \gamma, 0, \cos \gamma)$; 第二次旋转以后(图 2d), $O 、 B 、 C$ 和 $D$ 的坐标分别变成 $O(0,0,0) 、 B(0, \cos \beta, \sin \beta)$ 、 $C(\sin \gamma, \cos \beta, \cos \gamma+\sin \beta)$ 和 $D(\sin \gamma, 0, \cos \gamma)$ 。如果平面经
过原点, 则该平面方程可以表示为

$$
a x+b y+z=0
$$

代入坐标 $B 、 C$ 和 $D$, 得到楔形斜面 $O B C D$ 的平面 方程

$$
-\cot \gamma x-\tan \beta y+z=0
$$

矢量 $\boldsymbol{w}$ 和 $\boldsymbol{v}$ 可以表示为如下形式

$$
\begin{aligned}
& \boldsymbol{w}=\frac{-\cot \gamma \boldsymbol{i}-\tan \beta \boldsymbol{j}+\boldsymbol{k}}{\sqrt{1+\cot ^{2} \gamma+\tan ^{2} \beta}} \\
& \boldsymbol{v}=0 \boldsymbol{i}-\cos \beta \boldsymbol{j}+\sin \beta \boldsymbol{k} .
\end{aligned}
$$

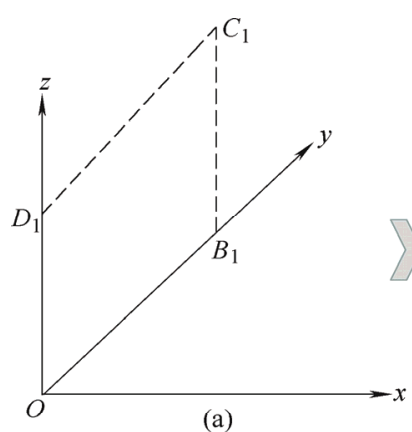

(a)
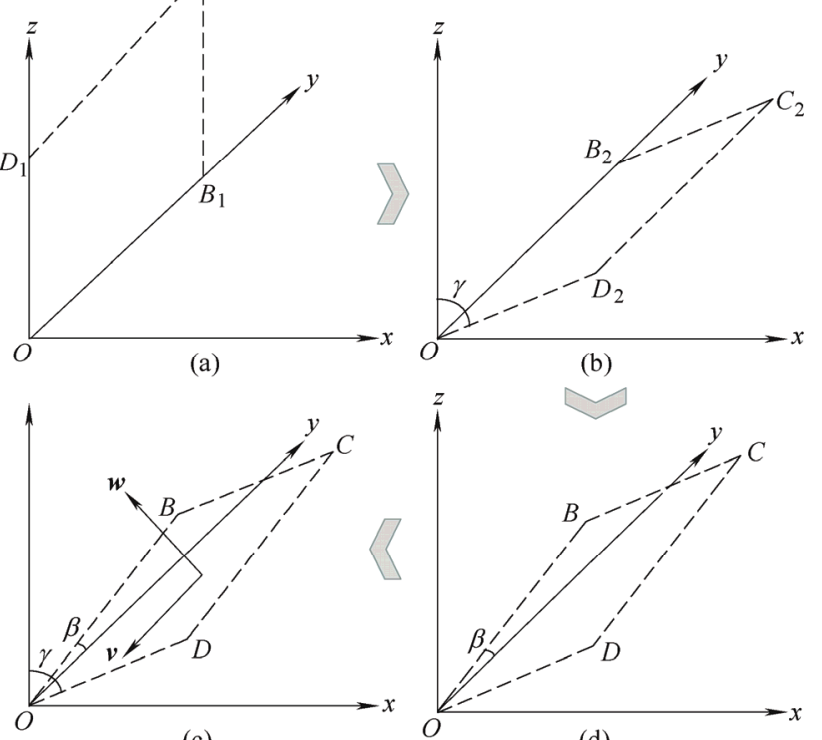

(d)

图 2 楔形斜面的局部几何形状表征

类似于普通螺纹连接 ${ }^{[26]}$, 楔形螺纹连接在拧紧 的过程中, 总的拧紧扭矩 $T$ 也包括 3 种扭矩形式

$$
T=T_{b}+T_{p}+T_{t}
$$

式中, $T_{b}$ 为端面摩擦扭矩; $T_{p}$ 为螺距扭矩; $T_{t}$ 为螺 纹面摩擦扭矩。

下面分别计算楔形螺纹连接的 3 种扭矩形式。 首先是端面摩擦扭矩 $T_{b}$, 如图 3 所示, 它可以通过 楔形锁紧螺母端面的摩擦力在端面接触区域 $A_{1}$ 上 的积分获得

$$
T_{b}=\iint_{A} u_{b} p_{b \Omega} r r \mathrm{~d} r \mathrm{~d} \theta
$$

式中, $\mu_{b}$ 为端面摩擦系数; $p_{b \Omega}$ 为端面压力分布; $r$ 为端面上的任意微小区域到中心点的距离; $\theta$ 为任意 微小区域相对初始位置的转角。进一步计算

$$
T_{b}=\iint_{A_{1}} u_{b} p_{b \Omega} r r \mathrm{~d} r \mathrm{~d} \theta=u_{b} \int \underbrace{\iint_{A_{1}} p_{b \Omega} r \mathrm{~d} r \mathrm{~d} \theta r}_{F} r=u_{b} F r_{b}
$$


式中, $F$ 为预紧力; $r_{b}$ 为端面等效半径, 可以通过 式(8)计算 ${ }^{[27]}$

$$
r_{b}=\frac{\int_{r_{b 1}}^{r_{b 2}} p_{b \Omega} r^{2} \mathrm{~d} r}{\int_{r_{b 1}}^{r_{b 2}} p_{b \Omega} r \mathrm{~d} r}
$$

式中, $r_{b 1}$ 为楔形锁紧螺母端面的最小半径; $r_{b 2}$ 为楔 形锁紧螺母端面的最大半径。当假设端面压力均匀 分布时, $r_{b}$ 的大小计算如下

$$
r_{b}=\frac{2}{3} r_{b 1} \frac{r_{b 2}^{2}+r_{b 1} r_{b 2}+r_{b 1}^{2}}{r_{b 1}^{2}+r_{b 1} r_{b 2}}
$$

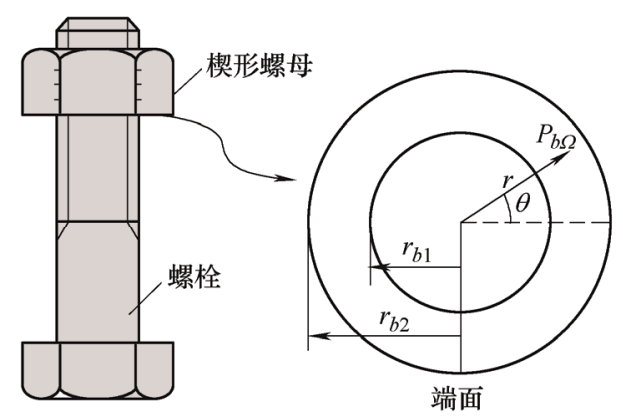

图 3 楔形锁紧螺母端面示意图

接着计算螺距扭矩 $T_{p}$ 和螺纹面摩擦扭矩 $T_{t}$ 的大 小。外螺纹牙尖和楔形斜面的接触配合是细长的螺 旋线接触, 螺旋线上的点到螺栓中心轴线的距离等 于螺纹公称直径的一半, 假设在拧紧过程中楔形斜 面的压力分布是 $p_{t \Omega}$, 则螺旋线上的法向压力和切向 摩擦力在轴线方向上的分力之和等于预紧力, 即

$$
F=\iint_{A_{2}}\left(p_{t \Omega} \boldsymbol{w} \cdot \boldsymbol{k}\right) \mathrm{d} s+\iint_{A_{2}}\left(-\mu_{t} p_{t \Omega} \boldsymbol{v} \cdot \boldsymbol{k}\right) \mathrm{d} s
$$

式中, $A_{2}$ 为牙尖和楔形斜面的接触区域; $\mu_{t}$ 为螺纹 面摩擦系数。将式(3)、(4)代入式(10)得到

$$
F=\iint_{A_{2}} p_{t \Omega} \mathrm{d} s \cdot\left(\frac{1-\mu_{t} \sin \beta \sqrt{1+\cot ^{2} \gamma+\tan ^{2} \beta}}{\sqrt{1+\cot ^{2} \gamma+\tan ^{2} \beta}}\right)
$$

因此

$$
\iint_{A_{2}} p_{t \Omega} \mathrm{d} s=F /\left(\frac{1-\mu_{t} \sin \beta \sqrt{1+\cot ^{2} \gamma+\tan ^{2} \beta}}{\sqrt{1+\cot ^{2} \gamma+\tan ^{2} \beta}}\right)
$$

通过楔形斜面的接触压力积分, 可以计算 $T_{p}$ 的大小

$$
T_{P}=\iint_{A_{2}}\left(-p_{t \Omega 2} \boldsymbol{w} \cdot \boldsymbol{j}\right) \frac{D}{2} \mathrm{~d} s=\iint_{A_{2}} p_{t \Omega} \mathrm{d} s \cdot \frac{\tan \beta D}{2 \sqrt{1+\cot ^{2} \gamma+\tan ^{2} \beta}}
$$

式中, $D$ 为螺纹的公称直径。将式(12)代入式(13), 得

$$
T_{P}=\frac{F \tan \beta D}{2\left(1-\mu_{t} \sin \beta \sqrt{1+\cot ^{2} \gamma+\tan ^{2} \beta}\right)}
$$

同理, 通过楔形斜面的切向摩擦力积分, 可以计算 $T_{t}$ 的大小

$$
T_{t}=\iint_{A_{2}}\left(-\mu_{t} p_{t \Omega} \mathbf{v} \cdot \mathbf{j}\right) \frac{D}{2} \mathrm{~d} s=\iint_{A_{2}} p_{t \Omega} \mathrm{d} s \cdot \frac{\mu_{t} \cos \beta D}{2}
$$

同样将式(12)代入式(15), 得

$$
T_{t}=\frac{F \mu_{t} \cos \beta D \sqrt{1+\cot ^{2} \gamma+\tan ^{2} \beta}}{2\left(1-\mu_{t} \sin \beta \sqrt{1+\cot ^{2} \gamma+\tan ^{2} \beta}\right)}
$$

最后, 将式(7)、(14)和(16)代入式(5)可以得到总拧 紧扭矩和预紧力之间的关系

$$
T=F\left(u_{b} r_{b}+\frac{D}{2} \frac{\tan \beta+\mu_{t} \cos \beta \sqrt{1+\cot ^{2} \gamma+\tan ^{2} \beta}}{1-\mu_{t} \sin \beta \sqrt{1+\cot ^{2} \gamma+\tan ^{2} \beta}}\right)
$$

式(17)就是楔形螺纹连接拧紧过程的扭拉关系理论 公式, 可以看出, 对于特定几何形状的楔形锁紧螺 母, 拧紧扭矩 $T$ 和预紧力 $F$ 之间呈线性关系。普通 螺纹连接的扭拉关系理论公式如式(18)所示 ${ }^{[26]}$

$$
T=F\left(\mu_{b} r_{b}+\frac{P}{2 \pi}+\frac{\mu_{t} r_{t}}{\cos \alpha}\right)
$$

式中, $P$ 为螺距; $\alpha$ 为一半牙型角; $r_{t}$ 为螺纹面等效 半径, 其大小可以通过假设螺纹面压力为均匀分布 近似计算。对比式(17)和(18)可以看出, 楔形螺纹连 接的扭拉关系理论公式更加复杂, 其中, 螺纹面摩 擦扭矩和螺距扭矩的计算公式有着显著差异, 同普 通螺纹连接相比, 考虑了楔形角度、牙型角以及公 称直径的影响。

\section{2 楔形螺纹连接扭拉关系理论公式}

的仿真验证

\section{1 楔形螺纹连接的有限元建模}

本节将建立楔形锁紧螺母和普通螺栓的精确 有限元模型, 施加拧紧扭矩, 仿真楔形锁紧螺母 的拧紧过程, 得到拧紧扭矩和预紧力之间的关系, 验证理论式(17)的可靠性。建模选取的螺纹规格是 公称直径 M10, 螺距 $1.5 \mathrm{~mm}$, 基于文献 ${ }^{[20]}$ 中关于 楔形螺纹轮廓的数学表达式, 采用 FUKUOKA 和 NOMURA ${ }^{[28]}$ 提出的螺纹网格划分方法, 利用前处 理软件 HYPERMESH ${ }^{\circledR} 12.0$ 建立楔形螺纹连接的 三维有限元模型, 如图 4a 所示, 该方法考虑了楔 
形螺纹和普通螺纹的精确螺旋形状, 并将所有网 格都划分成六面体, 大大提高了求解精度和求解 效率。

楔形螺纹连接的有限元模型包括楔形锁紧螺 母、普通螺栓和上被压件, 为了简化求解过程, 没 有建立下被压件的有限元模型, 而且螺栓头和螺母 都简化成圆柱体, 这种建模方式也被很多学者采 用 ${ }^{[4-6,20,29-31]}$, 其合理性和可靠性已经得到了广泛验 证。作为对比, 还建立了相同规格的普通螺纹连接 的有限元模型, 内外螺纹剖视图如图 4b 所示。

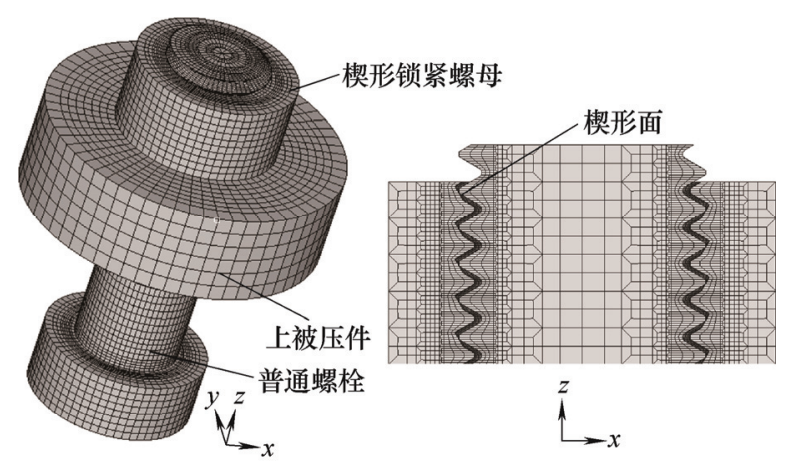

(a) 楔形螺纹连接

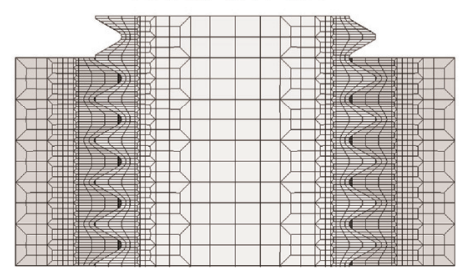

(b) 普通螺纹连接

图 4 楔形螺纹连接和普通螺纹连接的有限元模型

有限元模型的相关参数设置如表 1 所示, 采用 的实体单元类型是 SOLID 185, 材料是 8.8 级的 45 钢, 对应的弹性模量和泊松比分别是 $206 \mathrm{GPa}$ 和 0.3 , 不考虑材料塑性的影响。楔形螺纹连接的有限 元模型中有两对接触对: 螺纹接触对和端面接触对 (位于螺母和上被压件之间), 接触对包括接触面和 目标面, 接触面和目标面分别采用 CONTA173 单元 和 TARGE170 单元进行网格划分, 两对接触对的摩 擦因数均设为 0.15 。采用 MPC184 单元法施加拧紧 扭矩来产生预紧力, 如图 5 所示, 首先确定一个活 动节点, 将活动节点和螺母端面的节点通过 MPC184 单元耦合连接, 对活动节点施加一个拧紧 力矩就可以实现楔形锁紧螺母的拧紧过程。边界条 件的设置如下：螺栓头部和上被压件底面全约束。 为了保证有限元仿真结果的求解精度, 还对有限元 网格进行了密度测试, 最终确定有限元网格数量在 160000 左右。有限元求解过程均在 ANSYS16.0 ${ }^{\circledR}$ 中 进行。
表 1 有限元模型的参数设置

\begin{tabular}{cc}
\hline 参数 & 类型或数值 \\
\hline 单元类型 & SOLID 185 \\
弹性模量/ GPa & 206 \\
泊松比 & 0.3 \\
接触面 & CONTA173 \\
目标面 & TARGE170 \\
摩擦因数 & 0.15 \\
\hline
\end{tabular}

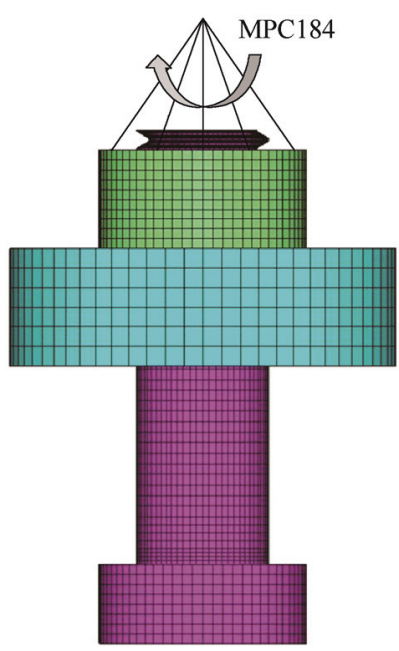

图 5 采用 MPC184 单元施加拧紧扭矩示意图

\section{2 楔形螺纹连接的拧紧过程仿真}

分别仿真楔形螺纹连接和普通螺纹连接的拧 紧过程, 获得离散的拧紧扭矩和预紧力数据点, 并 根据式(17)和(18)分别绘制楔形螺纹连接和普通 螺纹连接的扭拉关系直线, 如图 6 所示。从图中可 与看出, 有限元仿真得到的扭矩-预紧力数据点基 本上落在基于式(17)绘制的扭拉关系直线上，可 以初步验证楔形螺纹连接理论公式(式(17))的可靠 性; 此外, 在其他模型参数一致的情况下, 楔形螺 纹连接扭拉关系的直线斜率明显大于普通螺纹连接 扭拉关系的直线斜率，这也验证了之前学者们通过 试验研究发现楔形锁紧螺母比普通螺母更难拧紧的 结论 ${ }^{[17,22-23]}$ 。

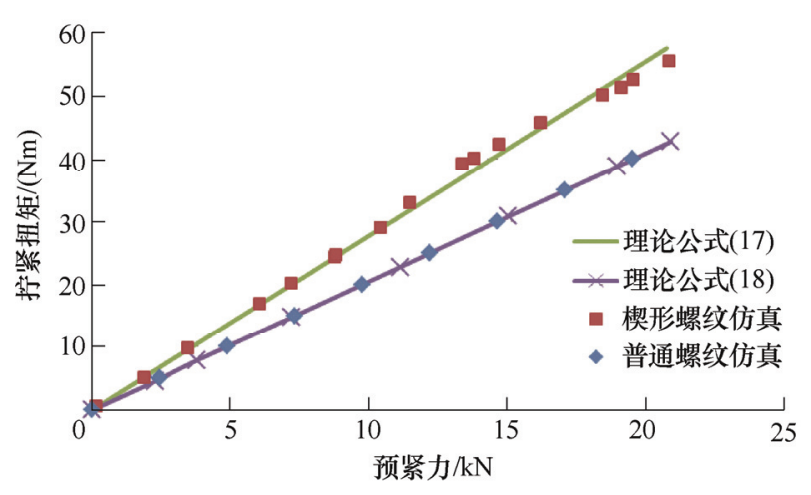

图 6 基于有限元仿真和理论公式的扭拉关系 


\section{3 楔形螺纹连接扭拉关系理论公式验证}

下面将改变不同的楔形螺纹连接参数, 同样采 用 MPC184 单元法施加拧紧扭矩仿真拧紧过程, 获得拧紧扭矩和预紧力的离散数据点, 对比式(17) 所绘制的扭拉关系直线, 进一步验证楔形螺纹连 接扭拉关系理论公式(式(17))的可靠性。首先, 改 变不同的端面摩擦因数和螺纹面摩擦因数, 如表 2 的前 9 行所示, 端面摩擦因数和螺纹面摩擦因数 分别是 $0.05 、 0.15$ 和 0.3 的任意两组组合, 共计 9 种组合。图 7 展示了在这 9 种摩擦因数组合的条 件下, 式(17)和有限元仿真所得到的楔形螺纹连接 扭拉关系, 从图中可以看出, 无论何种端面摩擦 因数和螺纹面摩擦因数组合, 有限元仿真得到的 扭矩-预紧力数据点基本上落在了基于式(17)所绘 制的扭拉关系直线上, 而且, 摩擦因数越大, 扭 拉关系直线的斜率也越大, 即楔形锁紧螺母将更 难拧紧。为了定量比较仿真结果和理论结果, 采 用最小二乘法对仿真得到的离散数据点进行线性 拟合, 并根据式(19)分别计算基于有限元仿真和理 论公式得到的扭矩系数 $K$

$$
T=K D F
$$

表 2 是计算得到的仿真和理论的扭矩系数大 小, 以及二者之间的偏差, 偏差通过式(20)计算 得到

$$
\text { Error }=\frac{\left|K_{1}-K_{2}\right|}{K_{1}}
$$

$K_{1}$ 表示根据理论式(18)计算的扭矩系数, $K_{2}$ 表 示根据仿真数据拟合得到的扭矩系数。从表 2 可以 看出, 理论和仿真的扭矩系数偏差非常小, 最大值 仅为 $1.92 \%$, 最小值为 $0.03 \%$ 。

接着, 改变不同的楔形角大小, 楔形锁紧螺 母的典型楔形角是 $30^{\circ}$, 分别建立楔形角是 $15^{\circ}$ 、 $20^{\circ} 、 25^{\circ} 、 35^{\circ} 、 40^{\circ}$ 和 $45^{\circ}$ 的楔形锁紧螺母, 如图 8 所示。在不同楔形角的条件下, 基于式(17) 和有限元仿真得到的楔形螺纹连接扭拉关系如 图 9 所示, 从图中可以看出, 所有的仿真数据点 基本上都落在了理论公式得到的扭拉关系直 线上, 而且, 随着楔形角增大, 扭拉关系直线的 斜率逐渐变小, 即楔形角越小, 楔形锁紧螺母越 难拧紧。从表 2 可以看出, 对于不同的楔形角, 基于式(17)得到的扭矩系数和有限元仿真拟合 的扭矩系数基本一致, 二者之间的最大偏差仅 为 $1.55 \%$ 。

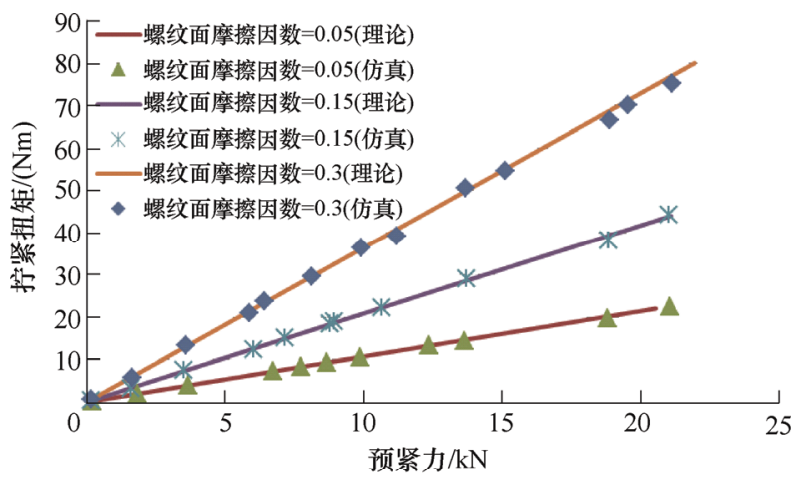

(a) 端面摩擦因数 $=0.05$

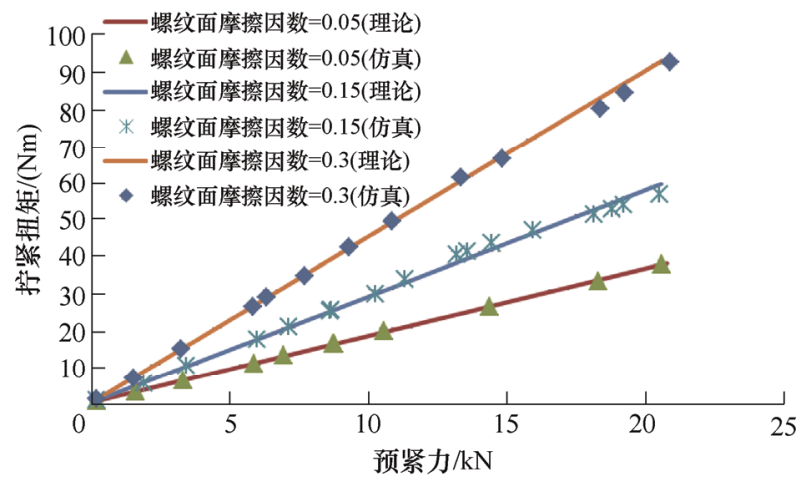

(b) 端面摩擦因数 $=0.15$

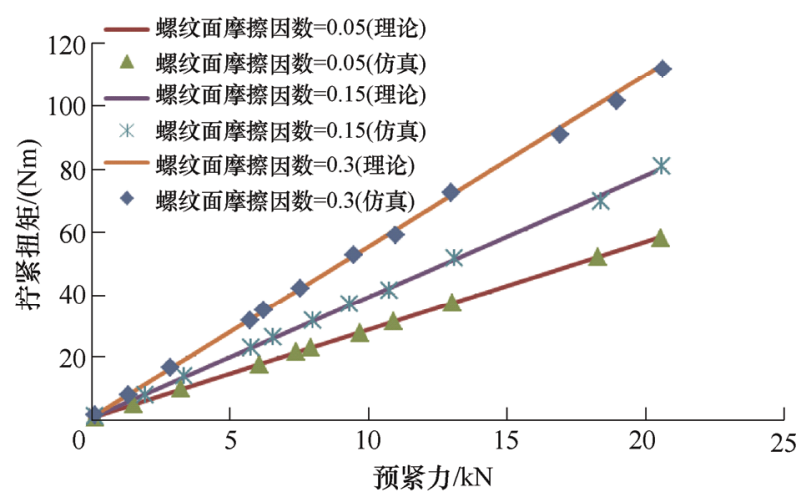

(c) 端面摩擦因数 $=0.3$

图 7 不同摩擦系数条件下基于理论公式和有限元 仿真的扭拉关系
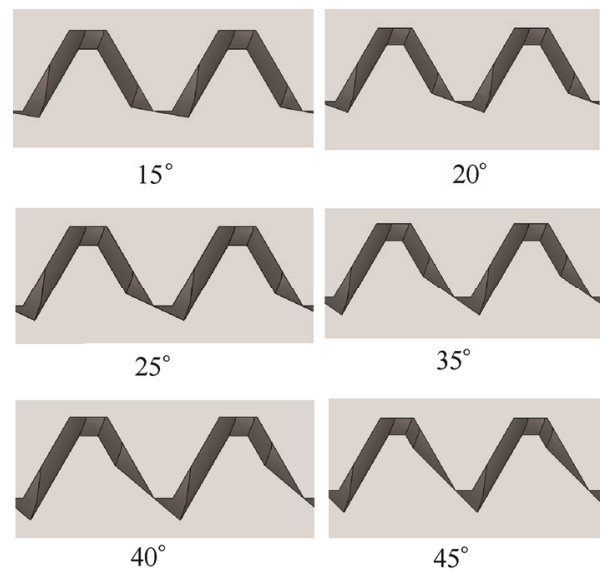

图 8 不同楔形角的楔形螺纹连接 


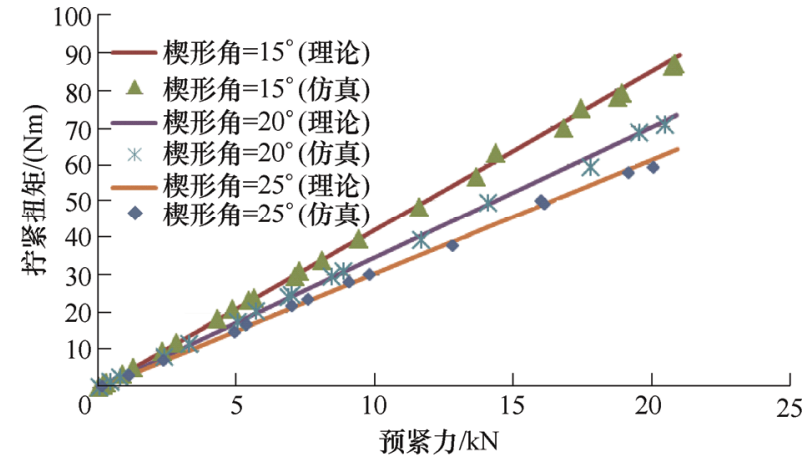

(a) 楔形角 $=15^{\circ} 、 20^{\circ}$ 和 $25^{\circ}$

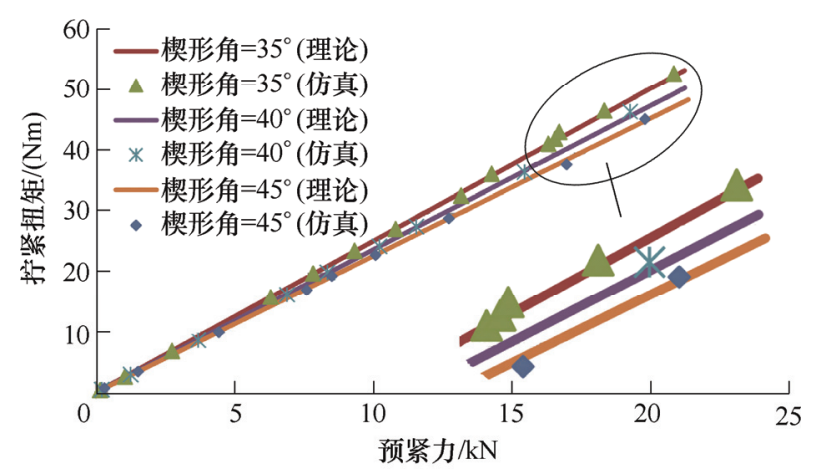

(b) 楔形角 $=35^{\circ} 、 40^{\circ}$ 和 $45^{\circ}$

图 9 不同楔形角条件下基于理论公式和有限元 仿真的扭拉关系

表 2 不同参数条件下扭矩系数的理论值和仿真值

\begin{tabular}{|c|c|c|c|c|c|c|c|}
\hline $\begin{array}{l}\text { 序 } \\
\text { 号 }\end{array}$ & $\begin{array}{l}\text { 端面 } \\
\text { 摩擦 } \\
\text { 因数 }\end{array}$ & $\begin{array}{c}\text { 螺纹 } \\
\text { 面摩 } \\
\text { 擦因 } \\
\text { 数 }\end{array}$ & $\begin{array}{c}\text { 楔形角 } \\
\text { / } \\
\left({ }^{\circ}\right)\end{array}$ & $\begin{array}{l}\text { 螺距 } \\
/ \mathrm{mm}\end{array}$ & $\begin{array}{l}\text { 扭矩系数 } \\
\text { (理论值) }\end{array}$ & $\begin{array}{c}\text { 扭矩系 } \\
\text { 数 } \\
\text { (仿真值) }\end{array}$ & $\begin{array}{l}\text { 偏差 } \\
(\%)\end{array}$ \\
\hline 1 & & 0.05 & & & 0.1078 & 0.1069 & 0.91 \\
\hline 2 & 0.05 & 0.15 & & & 0.2099 & 0.2098 & 0.03 \\
\hline 3 & & 0.3 & & & 0.3668 & 0.3606 & 1.68 \\
\hline 4 & & 0.05 & & & 0.1750 & 0.1748 & 0.11 \\
\hline 5 & 0.15 & 0.15 & 30 & 1.5 & 0.2771 & 0.2757 & 0.50 \\
\hline 6 & & 0.3 & & & 0.4339 & 0.4256 & 1.92 \\
\hline 7 & & 0.05 & & & 0.2758 & 0.2748 & 0.34 \\
\hline 8 & 0.3 & 0.15 & & & 0.3778 & 0.3764 & 0.38 \\
\hline 9 & & 0.3 & & & 0.5347 & 0.5266 & 1.52 \\
\hline 10 & & & 15 & & 0.4230 & 0.4170 & 1.43 \\
\hline 11 & & & 20 & & 0.3489 & 0.3435 & 1.55 \\
\hline 12 & & & 25 & & 0.3054 & 0.3023 & 1.01 \\
\hline 13 & 0.15 & 0.15 & 35 & 1.5 & 0.2573 & 0.2592 & 0.75 \\
\hline 14 & & & 40 & & 0.2428 & 0.2442 & 0.56 \\
\hline 15 & & & 45 & & 0.2320 & 0.2332 & 0.51 \\
\hline 16 & & & & 1 & 0.2682 & 0.2695 & 0.45 \\
\hline 17 & 0.15 & 0.15 & 30 & 1.25 & 0.2726 & 0.2720 & 0.23 \\
\hline
\end{tabular}

最后，改变不同的螺距大小，将螺纹螺距改变 成 $1 \mathrm{~mm}$ 和 $1.25 \mathrm{~mm}$, 在式(17)中表现为螺旋升角 $\beta$ 产生了相应变化, 图 10 展示了不同螺距条件下基于 式(17)和有限元仿真得到的扭拉关系, 从图中同样 可以看出, 有限元仿真得到的离散数据点也基本上 落在了基于理论公式所绘制的扭拉关系直线上。从 表 2 也可以看出, 理论和仿真的扭矩系数偏差最大 值仅为 $0.45 \%$ 。

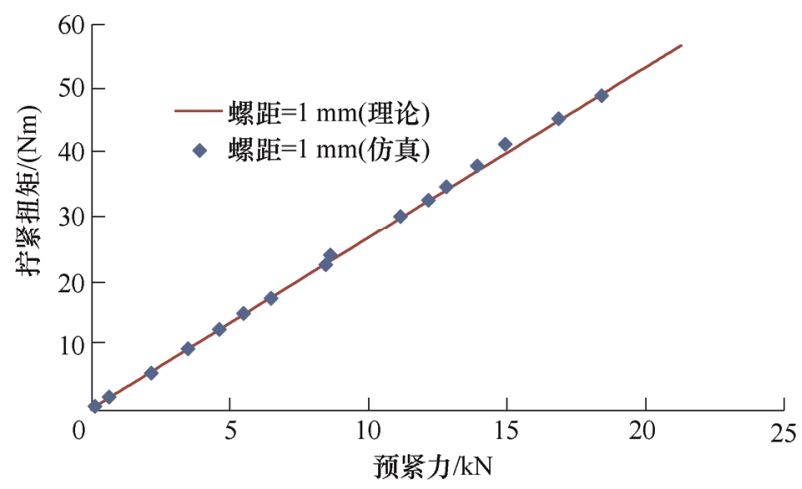

(a) 螺距 $=1 \mathrm{~mm}$

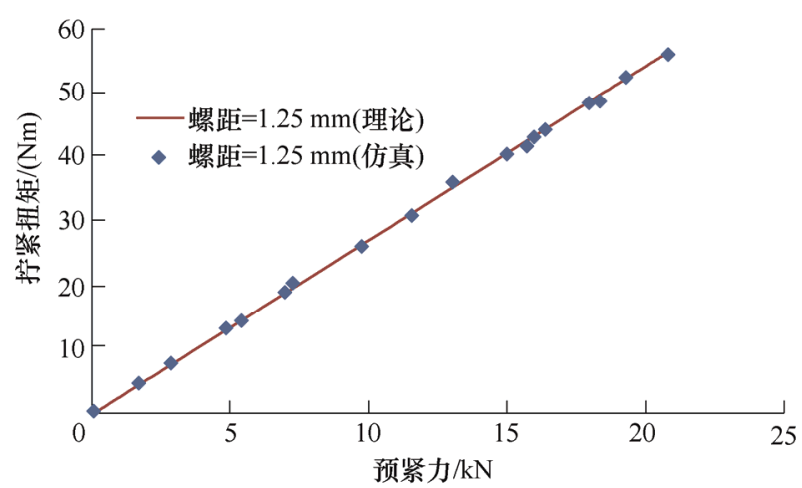

(b) 螺距 $=1.25 \mathrm{~mm}$

图 10 不同螺距条件下基于理论公式和有限元 仿真的扭拉关系

综上, 从上述的研究中可以发现, 无论改变 何种参数大小，基于有限元仿真得到的扭拉关系 和基于式(17)得到的扭拉关系基本吻合，二者之 间的最大偏差不超过 $2 \%$ ，这足以证明计算得到 的楔形螺纹连接扭拉关系理论公式(式(17)) 是可 靠的。

\section{3 楔形螺纹连接的拧紧试验研究}

\section{1 试验设计和试验方法}

选择的楔形锁紧螺母形式是六角法兰螺母和 游动双耳托板螺母, 如图 11 所示, 这两类楔形锁 紧螺母是航空航天领域广泛应用的楔形锁紧螺 母。首先, 通过拧紧试验进一步验证理论公式的 
可靠性, 由于实际的螺纹面摩擦系数和端面摩擦 系数很难直接准确地测量, 因此采用一种间接的 方式验证理论公式的可靠性。具体过程如下：选 择六角法兰螺母作为研究对象, 通过扭拉试验获 得总的拧紧扭矩 $T$ 和预紧力 $F$ 之间的关系曲线, 以及螺纹面扭矩+螺距扭矩 $\left(T_{t p}=T_{t}+T_{p}\right)$ 和预紧力之 间的关系曲线, 通过式(21)和(22)分别计算螺纹面 摩擦系数和端面摩擦系数

$$
\begin{gathered}
\mu_{t}=\frac{2 T_{t p}-\tan \beta \cdot D \cdot F}{\sqrt{1+\cot ^{2} \gamma+\tan ^{2} \beta}\left(\cos \beta \cdot D \cdot F+2 T_{t p} \sin \beta\right)} \\
u_{b}=\frac{T-T_{t p}}{F \cdot r_{b}}
\end{gathered}
$$

将这两个摩擦系数作为楔形螺纹连接拧紧仿真 的初始条件, 获得扭拉关系, 如果仿真得到的扭拉 关系和试验得到的扭拉关系基本吻合，则间接证明 了理论公式的可靠性。

接着, 为了研究不同因素对楔形锁紧螺母扭 拉关系和重复拧紧后紧固性能的影响规律, 根据 螺母形式、螺纹规格、螺母材料、螺栓材料和拉 板材料等因素的不同, 共设计了 6 组试验样本, 如表 3 所示。这 6 组试验样本需要进行两种试验, 第一种是扭拉关系试验, 计算楔形锁紧螺母重复 拧紧 5 次的扭矩系数大小, 每组有 5 个试验样件, 即重复试验 5 次; 第二种是重复拧紧的紧固性能 试验, 将 6 组试验样本重复拧紧 50 次, 获得第 50 次的拧紧扭矩和拆卸扭矩大小, 并分别计算拆卸 扭矩和拧紧扭矩的比值, 评估楔形锁紧螺母经过 多次重复拆装以后的紧固性能, 每组同样需要重 复试验 5 次。
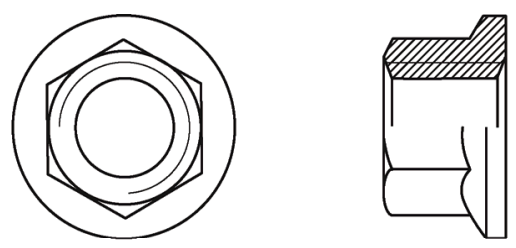

(a) 六角法兰螺母
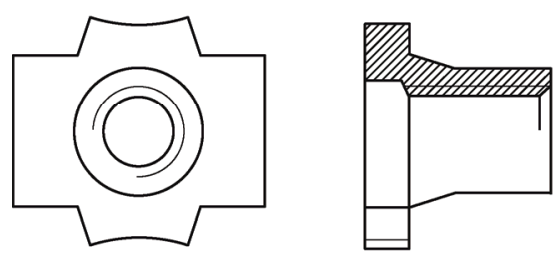

(b) 游动双耳托板螺母

图 11 两种楔形锁紧螺母形式

\begin{tabular}{|c|c|c|c|c|c|c|}
\hline 序号 & $\begin{array}{l}\text { 试验 } \\
\text { 名称 } \\
\end{array}$ & $\begin{array}{l}\text { 螺母 } \\
\text { 形式 }\end{array}$ & 规格 & 螺母材料 & 拉板材料 & 螺栓材料 \\
\hline 1 & \multirow{6}{*}{$\begin{array}{l}\text { 拧紧 } \\
\text { 试验 }\end{array}$} & & \multirow{4}{*}{ M10 } & TC4 & $20 \mathrm{Cr} 13$ & TC4 \\
\hline 2 & & $\begin{array}{l}\text { 六角 } \\
\text { 法兰 }\end{array}$ & & GH2132 & $20 \mathrm{Cr} 13$ & GH4169 \\
\hline 3 & & 螺母 & & $\mathrm{TC} 4$ & 钢摩擦片 & TC4 \\
\hline 4 & & \multirow{3}{*}{$\begin{array}{l}\text { 游动 } \\
\text { 双耳 } \\
\text { 托板 } \\
\text { 螺母 }\end{array}$} & & GH2132 & 钢摩擦片 & GH4169 \\
\hline 5 & & & \multirow{2}{*}{ M8 } & $1 \mathrm{Cr} 17 \mathrm{Ni} 2$ & zl205A & 1Cr17Ni2 \\
\hline 6 & & & & 1Cr17Ni2 & 铝 2219 & 1Cr17Ni2 \\
\hline
\end{tabular}

表 3 拧紧试验样本组成

拧紧试验通常在带有轴力扭矩复合传感器的扭 拉试验机上进行, 扭拉试验机的实物图如图 12a 所 示, 其工作原理如图 $12 \mathrm{~b}$ 所示。扭拉试验机由电机 经过谐波减速机减速后提供拧紧动力源, 在拧紧头 和减速机间安装扭矩传感器和角度编码器, 直接测 得拧紧和拆卸过程中的扭矩以及任何时刻的转角, 角度编码器、扭矩传感器以及拧紧头封装为一体。 扭拉试验机还配有一套复合传感器, 可以测量螺栓 拧紧和拆卸过程中的预紧力以及螺纹面摩擦扭矩, 该传感器安装在一个固定支架上。在试验时将试件 垫块安装到传感器支架的左侧, 可以保证试验过程 中支撑面摩擦条件与实际产品中一致, 将有内螺纹 的试件或螺母等安装在传感器右侧的夹具内, 夹具 通过圆柱销与复合传感器连接来传递扭矩, 实现螺 纹面摩擦扭矩的测量。具体的试验操作过程可以参 考国家标准 GB/T 16823.3-2010《紧固件扭矩一夹 紧力试验》 ${ }^{[32]}$ 。

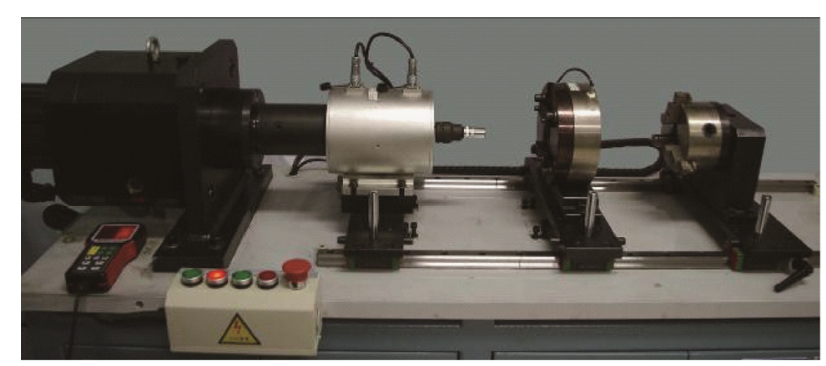

(a) 实物照片

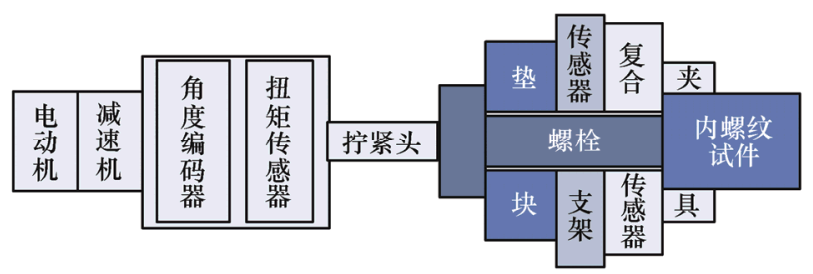

(b) 工作原理图

图 12 扭拉试验机

\section{2 扭拉关系理论公式的试验验证}

采用两个六角法兰螺母和普通螺栓连接作为试 
验验证样件, 螺纹规格是 M10, 螺距是 $1.5 \mathrm{~mm}$, 通 过扭拉试验获得总拧紧扭矩以及螺纹面扭矩+螺距 扭矩和预紧力之间的关系曲线, 如图 13 所示。根据 式(21)和式(22)分别计算两个样本的螺纹面摩擦系 数和端面摩擦系数: 经计算, 试件-1 的螺纹面摩擦 系数和端面摩擦系数分别是 0.2722 和 0.1724 ; 试 件-2 的螺纹面摩擦系数和端面摩擦系数分别是 0.1804 和 0.1766 。建立如图 5 所示的楔形螺纹连接 有限元模型, 将计算的螺纹面摩擦系数和端面摩擦 系数作为初始条件, 仿真拧紧过程, 得到总拧紧扭 矩和预紧力之间关系, 并和试验结果进行比较, 如 图 14 所示。从图中可以看出, 仿真得到的扭拉关系 离散数据点基本上落在了试验得到的扭拉关系曲线 上, 对试件的试验扭拉关系曲线和仿真离散数据点 进行线性拟合, 得到试件-1 的试验扭矩系数和仿真 扭矩系数分别是 0.4203 和 0.405 , 二者误差是 $3.78 \%$; 试件-2 的试验扭矩系数和仿真扭矩系数分 别是 0.3271 和 0.3291 , 二者误差是 $0.61 \%$, 这表明 试验得到的扭拉关系和仿真得到的扭拉关系的吻合 度非常高, 间接验证了楔形螺纹连接扭拉关系理论 公式的可靠性。

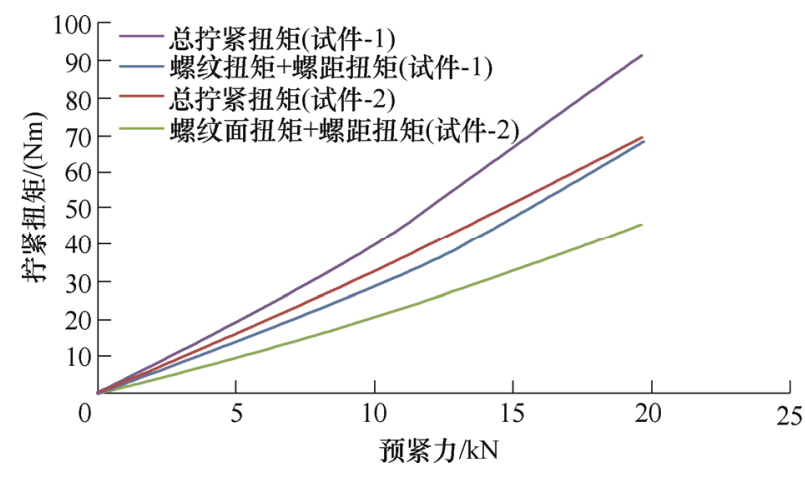

图 13 试验获得的总拧紧扭矩以及螺纹面扭矩+螺距扭矩和 预紧力之间的关系曲线

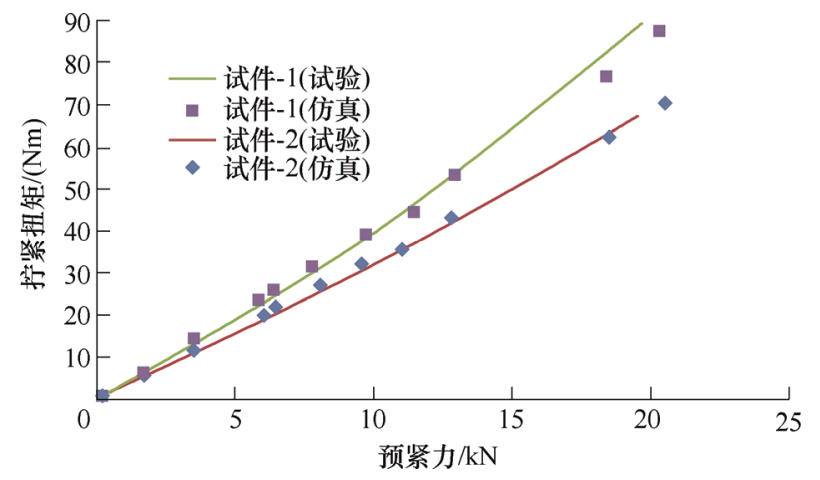

图 14 仿真和试验得到的扭拉关系

\section{3 扭拉关系的试验结果分析}

本节通过扭拉试验机对上述 6 组试验样本依次
进行扭拉试验, 根据试验结果得到扭矩-预紧力的数 据点, 采用最小二乘法对扭矩-预紧力数据点进行线 性拟合，根据拟合直线的斜率计算扭矩系数的大小， 图 15 展示了这 6 组试验样本在不同拧紧次数条件下 的扭矩系数变化趋势, 图 16 展示了这 6 组试验样本 的平均扭矩系数。从图中可以看出, 游动双耳托板 螺母的扭矩系数普遍高于六角法兰螺母, 即游动双 耳托板螺母在实际使用时更难拧紧, 这是因为游动 双耳托板螺母的结构比较特殊, 螺母体在支架中有 一定的活动量, 扭拉过程中摩擦条件复杂, 导致 $K$ 值变大。当螺栓和楔形锁紧螺母的材料是 TC4、拉 板材料是钢摩擦片时, 拧紧次数对扭矩系数基本没 有影响, 而其他样本的扭矩系数均随着拧紧次数的 增加而逐渐增大, 其中游动双耳托板螺母的扭矩系 数增大幅度最为明显。对于六角法兰螺母, 通过对 比样本 $1 、 2$ 和样本 $3 、 4$ 的试验结果, 发现螺母和 螺栓的材料类型对扭矩系数影响显著, 即材料是 GH2132 的六角法兰螺母和螺栓比材料是 TC4 的六 角法兰螺母和螺栓具有更大的扭矩系数, 可能因为 GH2132 的表面摩擦系数更大; 通过对比样本 $1 、 3$

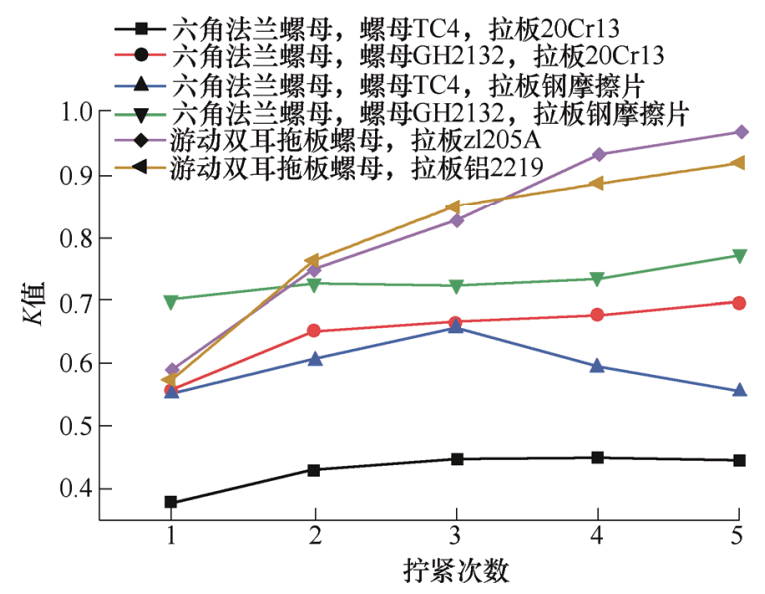

图 15 扭拉试验中各试验样本的 $K$ 值变化曲线

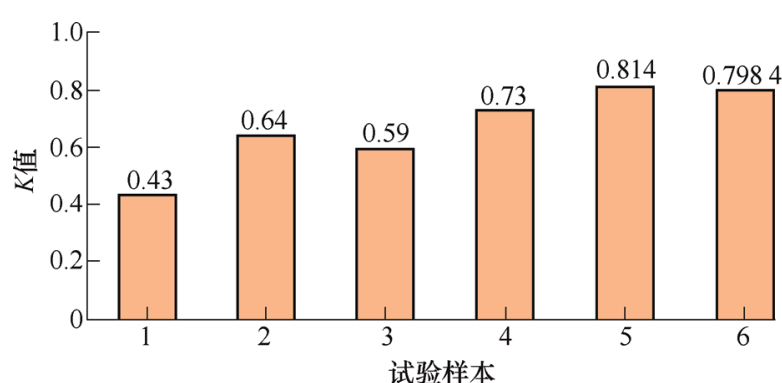

试验样本 1：六角法兰螺母, 螺母TC4, 拉板 $20 \mathrm{Cr} 13$ 试验样本2: 六角法兰螺母, 螺母 GH2132, 拉板 $20 \mathrm{Cr} 13$ 试验样本 3: 六角法兰螺母, 螺母TC4, 拉板钢摩擦片 试验样本 4: 六角法兰螺母, 螺母-GH2132, 拉板钢摩擦片 试验样本 5: 游动双耳托板螺母, 螺母 $1 \mathrm{Cr} 17 \mathrm{Ni} 2$, 拉板 $\mathrm{z} 1205 \mathrm{~A}$ 试验样本6: 游动双耳托板螺母, 螺母1Cr17 Ni2, 拉铝板 2219

图 16 扭拉试验中各试验样本的平均 $K$ 值大小 
和样本 2、4 的试验结果, 发现拉板的材料类型同样 对其扭矩系数影响显著, 即钢摩擦片的拉板比 $20 \mathrm{Cr} 13$ 的拉板具有更大的扭矩系数。对于游动双耳 托板螺母, 研究结果表明, 不同拉板材料对游动双 耳托板螺母的扭矩系数基本没有影响。

\section{4 重复拧紧性能的试验结果分析}

本节通过扭拉试验机对上述 6 组试验样本重复 拧紧了 50 次, 获得第 50 次的拧紧扭矩和拆卸扭矩 大小, 计算不同样本的拆卸扭矩和拧紧扭矩的比值, 如图 17 所示。从图中可以看出, 六角法兰螺母的扭 矩比值普遍高于游动双耳托板螺母的扭矩比值, 一 般比值越大, 表明其重复拧紧性能越好, 因此, 六 角法兰螺母在重复拧紧以后的紧固性能要优于游动 双耳托板螺母。对于六角法兰螺母, 从图中可以看 出, 样本 $3 、 4$ 得到的扭矩比值明显高于样本 $1 、 2$ 得到的扭矩比值, 其数值甚至超过了 $90 \%$, 这表明 当拉板材料是钢摩擦片时, 六角法兰螺母形式的楔 形螺纹连接具有非常优异的重复拧紧性能, 建议在 实际的工程应用中应该采用钢摩擦片的拉板结构; 而对于游动双耳托板螺母, 不同拉板材料的扭矩比 值基本相同，表明拉板的材料类型对游动双耳托板 螺母的重复拧紧性能几乎没有影响。

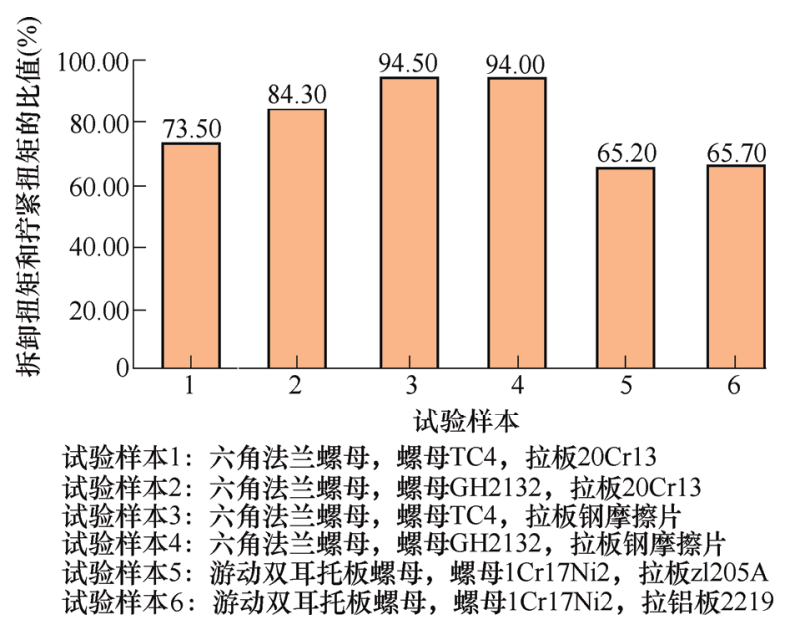

图 17 重复拧紧性能试验中各试验样本的拆卸扭矩和 拧紧扭矩的比值

\section{4 结论}

对楔形锁紧螺母的拧紧过程进行了理论分析、 仿真和试验验证, 在此基础上对航空航天领域的两 种典型楔形锁紧螺母的拧紧特性进行了试验研究, 得到了如下结论。

(1) 建立了楔形锁紧螺母拧紧扭矩和预紧力之 间的理论公式, 同普通螺纹连接相比, 提出的理论
公式考虑了楔形角度、牙型角以及公称直径的影响。

(2) 通过数值仿真和拧紧试验, 充分验证了楔 形螺纹连接扭拉关系理论公式的可靠性; 而且, 理 论和仿真结果均表明摩擦系数和螺距越大, 楔形角 越小, 楔形螺纹连接的扭矩系数将越大。

(3) 对于相同规格的普通螺纹连接和楔形螺纹 连接, 理论和仿真结果均表明, 在摩擦系数等参数 一致的情况下, 楔形螺纹连接具有更大的扭矩系数, 即楔形螺纹连接比普通螺纹连接更难拧紧。

(4) 扭拉试验的结果表明, 游动双耳托板螺母 的扭矩系数普遍高于六角法兰螺母, 对于六角法兰 螺母的楔形螺纹连接, 当螺栓螺母材料是 GH2132, 拉板材料是钢摩擦片时, 扭矩系数更大, 但材料类 型对游动双耳托板螺母的扭矩系数基本没有影响。

（5）重复拧紧性能的试验结果表明, 六角法兰 螺母的重复拧紧性能优于游动双耳托板螺母, 对于 六角法兰螺母的楔形螺纹连接, 当拉板材料是钢摩 擦片时, 其具有优异的重复拧紧性能, 而拉板的材 料类型对游动双耳托板螺母的重复拧紧性能几乎没 有影响。

\section{参 考 文 献}

[1] JUNKER G H. New criteria for self-loosening of fasteners under vibration[J]. SAE Transactions, 1969, 78: 314-335.

[2] BICKFORD J H. An introduction to the design and behaviour of bolted joints[M]. New York: Marcel Dekker Inc, 1995.

[3] PAI N G, HESS D P. Experimental study of loosening of threaded fasteners due to dynamic shear loads[J]. Journal of Sound and Vibration, 2002，253(3): 585-602.

[4] 杜永强, 刘建华, 刘学通, 等. 偏心载荷作用下螺栓连 接结构的松动行为研究 [J]. 机械工程学报, 2018, 46: 60-63.

DU Yongqiang, LIU Jianhua, LIU Xuetong, et al. Research on self-loosening behavior of bolted joints under eccentric excitation[J]. Journal of Mechanical Engineering, 2018, 46: $60-63$.

[5] IZUMI S, YOKOYAMA T, IWASAKI A, et al. Three-dimensional finite element analysis of tightening and loosening mechanism of threaded fastener[J]. Engineering Failure Analysis, 2005, 12(4): 604-615.

[6] 张明远, 鲁连涛, 唐明明, 等. 横向载荷作用下螺栓临 界松动载荷数值计算方法研究 [J]. 机械工程学报, 2018，54(5): 173-178. 
ZHANG Mingyuan, LU Liantao, TANG Mingming, et al. Research on numerical calculation method of critical load for bolt loosening under transverse loading[J]. Journal of Mechanical Engineering, 2018, 54(5): 173-178.

[7] 江文强, 墨泽, 安利强, 等. 考虑螺纹柔性的螺栓连接 临界松动载荷计算方法[J]. 机械工程学报, 2020, 56(15): 238-248

JIANG Wenqiang, MO Ze, AN Liqiang, et al. Computing method of bolted joint critical loosening load with flexible thread[J]. Journal of Mechanical Engineering, 2020, 56(15): $238-248$.

[8] 朱冀湘. 螺丝钉松动引发驾驶舱起火, 美国客机大西洋 上空惊魂[EB/OL]. [2010-06-30]. http: //gb.cri.cn/27824/ 2010/06/30/2585s2904522.htm.

ZHU Jixiang. An American passenger plane has been sent into a panic over the Atlantic after a loose screw caused a fire in the cockpit[EB/OL]. [2010-06-30] .http: //gb.cri.cn/ 27824/2010/06/30/2585s2904522.htm.

[9] IZUMI S, YOKOYAMA T, KIMURA $\mathrm{M}$, et al. Loosening-resistance evaluation of double-nut tightening method and spring washer by three-dimensional finite element analysis[J]. Engineering Failure Analysis, 2009, 16(5): 1510-1519.

[10] YOKOYAMA T, IZUMI S, SAKAI S. Loosening resistance evaluation of double-nut tightening method, spring washers, and conical spring washers finite element study $[C]$. Pressure Vessels and Piping Conference of the ASME. Chicago, 2008: 227-235.

[11] IZUMI S, KIMURA M, SAKAI S. Evaluation of loosening proof performance of plain washer and flange nut by three-dimensional finite element analysis[J]. Japan Society of Mechanical Engineers, 2006, 72(9) : 1292-1295.

[12] SASE N, NISHIOKA K, KOGA S, et al. An anti-loosening screw-fastener innovation and its evaluation[J]. Materials Processing Technologies, 1998, 77: $209-215$.

[13] SASE N, FUJII H. Optimizing study of SLBs for higher anti-loosening performance[J]. Materials Processing Technologies, 2001, 119: 174-179.

[14] RANJAN B S C, VIKRANTH H N, GHOSAL A. A novel prevailing torque threaded fastener and its analysis[J]. Journal of Mechanical Design, 2013, 135: 101007.

[15] BOETTCHER A. Ramp breaks common thread of fastener failures[J]. Journal of Mechanical Design, 2006, 78(19): $82-88$.

[16] 李作霖, 曾忠. 螺纹结构优化与防松应用研究[J]. 制造 业自动化，2011，33(12): 76-77.

LI Zuolin, ZENG Zhong. Structural optimization and the locking screw applied research[J]. Manufacturing Automation, 2011, 33(12): 76-77.

[17] 张翼, 吴云峰, 方红荣, 等. 楔形螺纹紧固件性能试验 研究 $[\mathrm{J}]$. 导弹与航天运载技术, 2020，372：123-126.

ZHANG Yi, WU Yunfeng, FANG Hongrong, et al. Experimental research on characters for wedging screw thread locknuts[J]. Missile and Space Launch Technology, 2020, 372: 123-126.

[18] 杨柳, 张雪峰, 间路. 楔型螺纹螺母可靠性初步评估方 法研究[J]. 宇航总体技术，2018，2(4): 31-35.

YANG Liu, ZHANG Xuefeng, YAN Lu. A preliminary evaluation of reliability for wedge screw nut[J]. Aerospace General Technology，2018， 2 (4): 31-35.

[19] 余威, 李建刚, 黄帅. 航空航天用 GH2132 系列楔形螺 纹托板螺母紧固件防松性能研究[J]. 宇航总体技术, 2018, 2(1): 62-70.

YU Wei, LI Jiangang, HUANG Shuai. Study on locking performance of GH2132 series wedge-shaped threaded bracket nut fasteners for aerospace[J]. Aerospace General Technology, 2018, 2(1): 62-70.

[20] LIU Jianhua, GONG Hao, DING Xiaoyu. Effect of ramp angle on the anti-loosening ability of wedge self-locking nuts under vibration[J]. Journal of Mechanical Design, 2018, 140(7): 072301-8.

[21] 李天雷, 李宏, 邓智昌, 等. 轴向预紧力对楔形螺母防 松性能的影响[J]. 矿山机械, 2018(46): 60-63.

LI Tianlei, LI Hong, DENG Zhichang, et al. Influence of axial pre-tightening force on anti-loosening performance of wedge nut[J]. Mining Machinery, 2018(46): 60-63.

[22] TREMSIN A S, YAU T Y, KOCKELMANN W. Non-destructive examination of loads in regular and self-locking spiralock ${ }^{\circledR}$ threads through energy resolved neutron imaging[J]. Strain, 2016, 52: 548-558.

[23] 魏天赐, 罗超. 使用 $30^{\circ}$ 楔形防松螺纹安装扭矩的建 议 [J]. 汽车实用技术, 2014(8): 88-89.

WEI Tianci, LUO Chao. Use $30^{\circ}$ wedge-locking threaded mounting torque suggestions $[\mathrm{J}]$. Automotive Practical Technology, 2014(8): 88-89.

[24] 上海底特精密紧固件有限公司. 美国施必牢防松螺母 及工具简介与产品标准 $[R]$. 上海: 上海底特, 2011 . 
Shanghai Detroit Precision Fastener Co., LTD. Spiralock anti-loosening nut of USA, tool introduction and product standard[R]. Shanghai: Shanghai Detroit, 2011.

[25] 张珊珊, 王健, 蔡明刚. 楔形防松螺纹的防松性能分 析[J]. 上海计量测试, 2018(3): 43-44.

ZHANG Shanshan, WANG Jian, CAI Minggang. Anti-loosing performance analysis of Locking threads with wedge ramp[J]. Shanghai Metrology and Testing, 2018(3): 43-44.

[26] LIU Jianhua, GONG Hao, DING Xiaoyu. Calculation of the effective bearing contact radius for precision tightening of bolted joints[J]. Advances in Mechanical Engineering, 2016, 8(9): 1-8.

[27] NASSAR S A, BARBER G C, ZUO D. Bearing friction torque in bolted joints[J]. Tribology Transactions, 2005, 48: $69-75$.

[28] FUKUOKA T, NOMURA M. Proposition of helical thread modeling with accurate geometry and finite element analysis[J]. Journal of Pressure Vessel Technology, 2008, 130(1): 011201.

[29] LIU Jianhua, GONG Hao, DING Xiaoyu. Study on the mechanism of preload decrease of bolted joints subjected to transversal vibration loading[J]. Journal of Engineering Manufacture, 2019, 233(12): 2320-2329.

[30] 巩浩, 刘检华, 丁晓宇, 等. 振动条件下螺纹预紧力衰
退机理和影响因素研究[J]. 机械工程学报, 2019, 55(11): 138-148.

GONG Hao, LIU Jianhua, DING Xiaoyu, et al. Study on the mechanism and influencing factors of preload decline for bolted joints under vibration[J]. Journal of Mechanical Engineering, 2019, 55(11): 138-148.

[31] JIANG Xiangjun, ZHU Yongsheng, HONG Jun. Investigation into the loosening mechanism of bolt in curvic coupling subjected to transverse loading[J]. Engineering Failure Analysis, 2013, 32: 360-373.

[32] 中华人民共和国国家质量监督检验检疫总局，中国国 家标准化管理委员会. GB/T 16823.3-2010 紧固件扭矩夹紧力试验[S]. 北京: 中国标准出版社, 2010 .

General Administration of Quality Supervision , Inspection and Quarantine of the People' $\mathrm{s}$ Republic of China, Standardization Administration of the People' $\mathrm{s}$ Republic of China. GB/T 16823.3-2010 fasteners torque/clamp force testing [S]. Beijing: Standards Press of China, 2010.

作者简介: 邓新建, 男, 1998 年出生。主要研究方向为螺纹高可靠性连 接和高性能装配技术。

E-mail: kevindxj@163.com

巩浩(通信作者), 男, 1992 年出生, 博士后。主要研究方向为高性能装 配、装配智能检测、螺纹高可靠性连接和松动失效机理。

E-mail: gongh0220@163.com 